\title{
Condicionantes de sucesso em projetos de software e sua influência nos resultados
}

Ralf Luis de Moura

Graduação em Ciência da Computação, pós-graduação em Desenvolvimento de Sistemas em Java (UFES) e mestrado em Engenharia Elétrica pela Universidade Federal do Espírito Santo. Doutorando em Administração na UFES, gerente de projetos de tecnologia na Vale S.A. e professor dos cursos superiores de Engenharia Elétrica, de Computação e de Controle e Automação, Sistemas de Informação e Tecnologia em Desenvolvimento de Sistemas, Redes de Computadores e Jogos Digitais nas Faculdades Integradas Espirito Santenses, Espirito Santo, Brasil ralfmoura@gmail.com

Teresa Cristina Janes Carneiro Doutorado em Administração e mestrado em Administração pelo Instituto COPPEAD/UFRJ. Graduação em Engenharia Civil pela UFES . Pesquisadora nas áreas de Sistemas de Informação, Tecnologia e Inovação, Administração Financeira e Administração Pública. Pró-Reitora de Administração da UFES . Professora do Departamento de Pós-Graduação em Administração da UFES, Espirito Santo, Brasil carneiro.teresa@gmail.com

Erica R. Freitas

Aluna de Pós-graduação em Gestão de Projetos da UFES, Espirito Santo, Brasil erica.r.freitas@gmail.com

Editor Científico: José Edson Lara

Organização Comitê Científico

Double Blind Review pelo SEER/OJS

Recebido em 07.02.2017

Aprovado em 03.12.2017 


\title{
Resumo
}

Projetos de software não têm atingido os índices de sucesso esperados e isso tem afetado as organizações de forma significativa. Esse trabalho analisou os fatores condicionantes de sucesso de projetos de softwares e seus impactos no sucesso do produto e no processo de desenvolvimento. Um modelo estrutural foi testado, em três cenários, por meio de uma pesquisa empírica realizada com profissionais de 77 empresas no Brasil. Os resultados sugerem que a Complexidade do Sistema, o Planejamento do Projeto e o Envolvimento da Equipe e dos Usuários influenciam o sucesso dos projetos de software. A Complexidade do Sistema é o fator com maior potencial de influência negativa, para o sucesso do projeto em geral e para quando apenas o sucesso do produto é testado, o Planejamento do Projeto tem maior influência positiva quando apenas o sucesso do processo de desenvolvimento é testado. Como contribuição esse estudo oferece um conjunto consolidado e parcimonioso de condicionantes do sucesso para projetos de software além de indicar os fatores que podem ter ações de acompanhamento e monitoramento priorizadas visando influenciar positivamente os resultados dos projetos.

Palavras-chave: Projetos; Desenvolvimento de Software; Condicionantes de Sucesso.

\section{Determinants of success in software projects and their influence on results}

\begin{abstract}
Software projects have not reached the expected success rates and this has significantly affected organizations. This work analyzed the success conditioning factors of software projects and their impact on the success of the product and the development process. A structural model was tested in three scenarios through an empirical research conducted with professionals from 77 software development companies in Brazil. The results suggest that System Complexity, Project Planning, and Team and User Involvement influence the success of software projects. System Complexity is the factor with the greatest potential of negative influence in the general success and when only product success is tested, Project Planning has greater positive influence when only the success of the development process is tested. As contribution, this study offers a consolidated and parsimonious set of specific success factors for software projects also indicate the factors that may have prioritized follow-up and monitoring actions aimed at positively influencing the results of projects.
\end{abstract}

Keywords: Projects; Software Development; Success Conditioners.

\section{Condicionantes de éxito en proyectos de software y su influencia en los resultados}




\section{Resumen}

Los proyectos de software no han sido incorporados a los índices de éxito esperados y han sido aceptados como organizaciones de forma significativa. Este trabajo analiza los factores condicionantes de éxito de proyectos de software y sus impactos en el éxito de producto y no proceso de desarrollo. Un modelo estructural para testado, en tres escenarios, por medio de una investigación empírica realizada con profesionales de 77 empresas no Brasil. Los resultados sugieren que la Complejidad del Sistema, el Planeamiento del Proyecto y el Envolvimiento de la Persona y los Usuarios influyen en el éxito de los proyectos de software. La Complejidad del Sistema es el factor con el mayor potencial de influencia negativa en el general éxito y en el éxito en el producto, el Planeación del Proyecto con mayor influencia positiva cuando el éxito del proceso de desarrollo es probado. Como contribución este estudio se propone un conjunto consolidado y condicional de condicionantes de éxito para los proyectos de software además de indicar los factores que pueden ser acciones de seguimiento y supervisión de priorizar visando influenciar positivamente los resultados de los proyectos.

Palabras clave: Proyectos; Desarrollo de Software; Acondicionadores de Éxito

\section{Introdução}

Projetos são empreendimentos temporários criados para operacionalizar as estratégias organizacionais sendo, portanto, uma das formas de as organizações atingirem seus objetivos estratégicos (Anantatmula, 2015). Devido às rápidas mudanças que ocorrem nos ambientes externos às organizações e à necessidade de acompanhar essas transformações, percebe-se um aumento do número de projetos em andamento nas organizações e a necessidade de desenvolver melhores práticas de gestão dessas ações. Em 2010, mais de 20\% das atividades econômicas globais foram realizadas por meio de projetos. Em economias emergentes esse montante excedeu a 30\% (Bredillet, 2010; Moura, 2015).

Segundo Kerzner (2010), com o passar dos anos os projetos têm se tornado mais complexos, motivo pelo qual as empresas passaram a reconhecer a importância da Gestão de Projetos que envolve o planejamento, a programação e o controle de uma série de atividades e tarefas integradas de tal maneira a atingir os objetivos pré-estabelecidos. 
Os softwares integram cada vez mais a agenda das organizações e os resultados dos projetos de desenvolvimento de softwares exercem importante influência sobre o sucesso das organizações (Aubry \& Hobbs, 2011; Schilling \& Hill, 1998). Os softwares têm características singulares que os tornam especialmente adequados para serem desenvolvidos por meio de projetos: são criados na maior parte das vezes para uso exclusivo e seu processo de desenvolvimento difere dos processos de fabricação tradicionais (Pressman, 2011, p.4), pelo fato de serem construídos na maioria das vezes sob medida e por passarem por um processo de desenvolvimento em fases.

O sucesso em um projeto significa o atendimento dos objetivos préestabelecidos. Porém, a medição de sucesso em projetos não é considerada trivial; depende de quem esteja analisando e do momento da análise (Cleland, 1999). Existe um relativo consenso quanto a algumas medidas de sucesso em projeto como, por exemplo, custo, qualidade, escopo e tempo, conhecida como a tripla restrição, porém muito se discute se estas medidas cobrem todas as dimensões do sucesso (Kerzner, 2010; De Wit, 1988; Cooke-Davies, 2002).

Pesquisadores estudam há mais de três décadas os motivos que podem levar os projetos ao sucesso e muitas são as tentativas de identificar os condicionantes de sucesso, também conhecidos como fatores críticos de sucesso (Dvir, Lipovetsky, \& Tishler, 1998). Estudo mundial do Project Management Institute [PMI] (2015) aponta que em média $36 \%$ dos projetos são considerados insucessos. Pesquisa da Standish Group (2015) mostra que a média histórica de sucessos em projetos de software é da ordem de $29 \%$. Projetos fracassados somados aos projetos entregues com limitações de escopo, de orçamento ou de prazo somam $71 \%$ do total.

Mediante estas constatações surgem as seguintes questões: Quais são os fatores condicionantes do sucesso de projetos de desenvolvimento de software? Quais fatores tem maior influência no sucesso dos projetos de software? Esse trabalho tem por objetivo analisar os fatores condicionantes de sucesso de projetos de softwares e seus impactos no sucesso do produto e no processo de desenvolvimento de software. Para tal, o trabalho consolidará, por meio de uma pesquisa bibliográfica, os condicionantes do sucesso de projetos de software e os critérios para se avaliar o sucesso destes projetos. Um modelo será proposto e testado empiricamente por meio de uma pesquisa em empresas de desenvolvimento de software no Brasil. 
Os resultados deste trabalho contribuem para a academia gerando um conjunto consolidado e parcimonioso de condicionantes do sucesso específicos para projetos de software que podem servir de referências para trabalhos futuros no campo dos estudos relacionados a projetos de software e para as organizações identificando os principais elementos que influenciam o resultado de seus projetos.

\section{Revisão da Literatura}

\subsection{Sucesso em Projetos de Desenvolvimento de Software}

Os projetos de software apresentam especificidades principalmente por gerarem produtos intangíveis e pela dificuldade do seu acompanhamento, além da dificuldade de comunicação entre os executores e os clientes /usuários (Prado, 1999). As etapas de desenvolvimento seguem um ciclo de vida com fases próprias, tais como a especificação de requisitos, análise, projeto, implementação, testes e implantação (Pressman, 2011; Moura, 2015).

Muitos projetos falham em atender às expectativas dos clientes e por isso muito se discute sobre o sucesso de projetos. Todavia, existe um consenso de que a determinação de falha ou sucesso de um projeto é considerada uma tarefa complexa (Mills \& Mercken, 2002; Cleland, 1999). Davis (2014) afirma que a percepção de sucesso pode variar para diferentes stakeholders e que não há um consenso sobre quais fatores determinantes do sucesso são relevantes.

Muito se discute sobre os melhores critérios para se medir o sucesso de projetos (Anantatmula, 2015; Moura \& Carneiro, 2015). Diversos pesquisadores analisaram os critérios de sucesso de projetos (De Wit, 1988; Atkinson, 1999; Lim \& Mohamed, 1999; Chan, 2001; Cooke-Davies, 2002; Kerzner, 2010; Anantatmula, 2015). Alguns critérios são objetivos, de fácil mensuração. Outros são subjetivos, mais difíceis de mensurar (Freeman \& Beale, 1992). Aspectos mais subjetivos que envolvem impactos de longo prazo e satisfação dos stakeholders são relacionados ao sucesso do produto, enquanto os aspectos técnicos relacionados a tripla restrição - escopo, qualidade, custo e prazo - são associados ao sucesso do processo de gestão do projeto (Cooke-Davies, 2002). A Tabela 1 consolida os atributos de 
sucesso de projetos de software identificados na revisão de literatura, as dimensões produto e processos foram propostas por Cooke-Davies (2002).

\section{Tabela 1}

Medidas de Sucesso em Projetos de Software

\begin{tabular}{|c|c|c|c|c|}
\hline Seq. & Dimensão & Indicador & Indicadores de Sucesso & Referências \\
\hline 1 & \multirow{5}{*}{ Produto } & F5a & Confiabilidade & \multirow{5}{*}{$\begin{array}{l}\text { Wallace, Keil e Rai } \\
\text { (2004); Cooke-Davies, } \\
\text { (2002); DeLone e } \\
\text { McLean (1992); } \\
\text { Freman e Beale (1992) }\end{array}$} \\
\hline 2 & & F5b & Manutenibilidade & \\
\hline 3 & & F5c & Satisfação dos usuários & \\
\hline 4 & & F5d & $\begin{array}{l}\text { Cumprimento das expectativas de tempo de } \\
\text { resposta }\end{array}$ & \\
\hline 5 & & F5e & $\begin{array}{l}\text { Cumprimento das expectativas de facilidade de } \\
\text { uso }\end{array}$ & \\
\hline 6 & \multirow{4}{*}{ Processo } & F6a & Cumprimento da Qualidade & \multirow{4}{*}{$\begin{array}{l}\text { Ika (2009); Kerzner } \\
\text { (2010); Atinkson } \\
\text { (1999); Cooke-Davies } \\
\text { (2002); De Wit (1988), } \\
\text { Anantatmula (2015); } \\
\text { Lim e Mohamed } \\
\text { (1999); Chan (2001) }\end{array}$} \\
\hline 7 & & F6b & $\begin{array}{l}\text { Cumprimento dos requisitos funcionais } \\
\text { (escopo) }\end{array}$ & \\
\hline 8 & & F6c & Cumprimento do prazo & \\
\hline 9 & & F6d & Cumprimento do custo & \\
\hline
\end{tabular}

Fonte: elaborada pelos autores (2016).

Esse estudo utilizou os indicadores apresentados na Tabela 1 na composição do modelo teórico de pesquisa. Os indicadores de sucesso da dimensão Produto representam os aspectos relacionados ao produto final e são específicos para o produto de software; os demais, relacionados à dimensão Processo, são aspectos do processo de desenvolvimento do produto de software e são aplicáveis a qualquer tipo de projeto. Estes atributos foram consolidados nos trabalhos de Wallace, Keil e Rai (2004), Cooke-Davies (2002), DeLone(1992), Ika (2009), Kerzner (2010) e Atinkson (1999).

A confiabilidade do software é avaliada pelo grau de estabilidade no funcionamento do software na percepção do usuário ou do desenvolvedor. O software é confiável quando durante sua operação apresenta um número reduzido de defeitos que não comprometem a sua disponibilidade (Pressman, 2011). A manutenibilidade é medida pela facilidade em realizar atualizações e correções do software; o cumprimento de requisitos funcionais envolve a constatação de que o software possui todas as funcionalidades acordadas inicialmente com os usuários; a satisfação dos usuários é avaliada pelo atendimento das expectativas do usuário quanto ao software desenvolvido; o tempo de resposta é relacionado ao tempo que o software leva para atender às necessidades de informação do usuário e, finalmente, a facilidade de uso é a percepção do usuário sobre o esforço do usuário 
para utilizar o software (Wallace, Keil, \& Rai, 2004). Estes indicadores compõem a dimensão Produto.

O cumprimento da Qualidade, escopo, prazo e custo são fatores tradicionais que fazem parte do conhecido triângulo de ferro ou tripla restrição, chamados assim por relacionarem as três restrições inter-relacionadas que determinam o sucesso dos projetos (Ika, 2009). O cumprimento destes aspectos atesta que o processo de desenvolvimento do software entregou o escopo acordado previamente e atendeu os requisitos de prazo, custo e qualidade definidos o plano do projeto de software (PMl, 2013). Estes indicadores compõem a dimensão Processo.

\subsection{Fatores Condicionantes de Sucesso de Projetos}

Vários são os fatores que podem comprometer o sucesso dos projetos. Pesquisadores relacionam o insucesso de projetos à complexidade crescente dos produtos (Reel, 1999; Kunz, 1989; Amrit et al., 2012). Outros pesquisadores atribuem o insucesso ao gerenciamento inadequado, falhas no gerenciamento de risco e comunicação inadequada durante a execução do projeto (Pressman, 2011; Brown, 1996; Royce, 1998; Barros, 2001; Taimour, 2005), além de fatores vinculados ao estágio de maturidade em gerenciamento de projeto da organização (Gray, 2001; Bouer \& Carvalho, 2005; Cooke-Davies, 2002; Moura, 2015).

Reel (1999), Kunz (1989) e Amrit (2012) vinculam possíveis insucessos à complexidade dos projetos e à forma de lidar com essa complexidade, falta ou excesso de preciosismo da equipe do projeto, organização da equipe, metodologias ultrapassadas, falhas no controle de qualidade e no gerenciamento do projeto. Charette (2005), Kaur e Sengupta (2011) citam ainda: requisitos incompletos, estimativa incorreta de tempo, mudanças no escopo, falhas em testes, baixa qualidade de gerenciamento e pressões comerciais.

Taimour (2005) afirma que o planejamento inadequado, objetivos pouco claros, objetivos que mudam durante o projeto, estimativas de tempo e de recursos pouco realistas, falta de suporte da alta direção, falhas na comunicação e perfis dos profissionais inadequados impactam negativamente o sucesso dos projetos. Brown (1996), Royce (1998), Goathan (2009), Barros (2001), Taimor (2005) e Pressman (2011) citam falhas na tomada de decisão, comunicação, gerenciamento de riscos e 
incertezas e falhas no gerenciamento da complexidade dos projetos como aspectos que têm impacto direto no sucesso dos projetos.

Os fatores relatados são relacionados a projetos em geral, não diferenciando qual o domínio ou o tipo de atuação do projeto. Para projetos de softwares, autores são mais específicos quando enumeram os fatores que podem influenciar o sucesso dos projetos (Wallace, Keil, \& Rai, 2004). Muito ainda se discute sobre isso (Moura, 2015) e dependendo do autor, diferentes fatores são apontados. Para esse estudo, optou-se por buscar os fatores apontados, em trabalhos teóricos e empíricos na literatura. $O$ resultado da coleta dos fatores condicionantes de sucesso de projetos está apresentado na Tebela 2. As dimensões apontadas foram criadas em função das características dos fatores identificados na revisão de literatura.

A dimensão Complexidade do Software representa a dificuldade para a execução do projeto, incluindo todas as fases do processo de desenvolvimento. Essa dimensão é composta por três subfatores: o subfator Ferramentas e Padrões de Qualidade do Projeto, que remete aos ambientes integrados de desenvolvimento (IDE) e aos padrões de qualidade aplicados ao projeto do software; o subfator Complexidade dos Requisitos, que envolve a dificuldade na compreensão e na implementação dos requisitos; e o subfator Novidade, que engloba o quanto de inovação será necessário no desenvolvimento do software (Wallace, Keil, \& Rai, 2004, Baccarini, Salm, \& Love, 2004, Barki et al., 1993; Kemerer \& Sosa, 1988; McFarlan, 1981; Schmidt et al., 2001).

A dimensão Envolvimento da Equipe e dos Usuários está vinculada ao relacionamento com os futuros usuários do software e aos aspectos das equipes e pessoas envolvidas no projeto, tais como o comprometimento e dedicação da equipe às metas do projeto; a disponibilidade dos usuários durante a fase de desenvolvimento do projeto e o comprometimento destes usuários com o sucesso do projeto (Abdel-Hamid, 1989; Alter \& Ginzberg, 1978; Jiang, Klein, \& Means, 2000; Schmidt et al., 2001; Wallace, Keil, \& Rai, 2004; Wallace, Keil, \& Rai, 2004a; Robey \& Farrow, 1982; Tait \& Vessey, 1988).

A dimensão Influências Econômicas e Ambientais representa questões externas ao projeto relacionadas à estabilidade do ambiente onde o projeto está sendo desenvolvido. Envolve aspectos de financiamento, questões legais e políticas e aspectos relacionadas à organização onde o software será implementado. Os subfatores que compõem essa dimensão são: Inflação e câmbio, Ambiente 
Organizacional e Leis e Regulamentos. Inflação e Câmbio estão relacionados a eventos econômicos externos que podem impactar os custos do projeto; o Ambiente Organizacional está relacionado a questões culturais e estruturais da organização da qual o projeto faz parte e que podem, de certa forma, influenciar o andamento do projeto e, finalmente, as Leis e Regulamentos relacionados ao objeto do software, que podem ser criados e/ou modificados no andamento do projeto, provocando mudanças (Dey, 2002; Mustafa \& Al-Bahar, 1991; Ewusi-Mensah \& Przasnyski, 1991; Jarvenpaa \& Ives, 1991; Jones, 1994; Wallace, Keil, \& Rai, 2004; Wallace, Keil, \& Rai, 2004a).

A dimensão Planejamento do Projeto refere-se a questões relacionadas ao alinhamento, planejamento e comunicação do projeto. A metodologia escolhida para o desenvolvimento do projeto, ágil ou tradicional, o ciclo de vida de desenvolvimento do software, a composição do time de projeto, a dispersão geográfica do time do projeto, a definição de responsabilidades dos envolvidos no projeto e a gestão destes envolvidos compõem esta dimensão Elkington \& Smallman, 2000); Baccarini, Salm \& Love, 2004).

Projetos de software podem ser influenciados pelos mesmos condicionantes que os demais projetos. Os itens enumerados na Tabela 2 são as referências utilizadas na pesquisa empírica detalhada posteriormente neste trabalho.

\section{Tabela 2}

Fatores condicionantes de sucesso de projetos

\begin{tabular}{|c|c|c|c|}
\hline Dimensão & Indicador & Variáveis & Referências \\
\hline \multirow[t]{3}{*}{$\begin{array}{l}\text { Complexidade do } \\
\text { Software }\end{array}$} & F1a & $\begin{array}{l}\text { Ferramentas e Padrões } \\
\text { de Qualidade }\end{array}$ & \multirow{3}{*}{$\begin{array}{l}\text { Warkentin et al (2009); Dey (2002); } \\
\text { Elkington e Smallman (2000); } \\
\text { Baccarini, Salm e Love (2004); } \\
\text { Grimsey e Lewis (2002); Costa, } \\
\text { Barros e Travassos (2007); Barki et al. } \\
\text { (1993); Kemerer e Sosa (1988); } \\
\text { McFarlan (1981); Schmidt et al. } \\
\text { (2001); Wallace, Keil, Rai (2004, } \\
\text { 2004a); Reel (1999); Kunz (1989); } \\
\text { Amrit et al. (2012); Charette (2005); } \\
\text { Kaur e Sengupta (2011) }\end{array}$} \\
\hline & F1b & $\begin{array}{l}\text { Complexidade de } \\
\text { Requisitos }\end{array}$ & \\
\hline & F1c & Novidade do Projeto & \\
\hline \multirow[t]{4}{*}{$\begin{array}{l}\text { Envolvimento da } \\
\text { Equipe e dos Usuários }\end{array}$} & $\mathrm{F} 2 \mathrm{a}$ & $\begin{array}{l}\text { Comprometimento da } \\
\text { equipe }\end{array}$ & \multirow{4}{*}{$\begin{array}{l}\text { Kloppenborg (2007); Nieto-Marote e } \\
\text { Vila (2010); Wallace, Keil e Rai } \\
\text { (2004, 2004a); Costa, Barros e } \\
\text { Travassos (2007); Elkington e } \\
\text { Smallman (2000); Robey e Farrow } \\
\text { (1982); Tait e Vessey (1988); Aundhe } \\
\text { e Saji (2009); Taylor (2006); Abdel- } \\
\text { Hamid e Madnick (1989); Alter e } \\
\text { Ginzberg (1978); Jiang, Klein e }\end{array}$} \\
\hline & $\mathrm{F} 2 \mathrm{~b}$ & $\begin{array}{l}\text { Dedicação dos } \\
\text { Recursos ao Projeto }\end{array}$ & \\
\hline & $\mathrm{F} 2 \mathrm{c}$ & $\begin{array}{l}\text { Disponibilidade dos } \\
\text { Usuários }\end{array}$ & \\
\hline & $\mathrm{F} 2 \mathrm{~d}$ & $\begin{array}{l}\text { Comprometimento dos } \\
\text { Usuários }\end{array}$ & \\
\hline
\end{tabular}




\begin{tabular}{|c|c|c|c|}
\hline Dimensão & Indicador & Variáveis & Referências \\
\hline & & & $\begin{array}{l}\text { Means (2000); Schmidt et al. (2001); } \\
\text { Dhar e Balakrishnnan (2006); } \\
\text { Warkentin et al. (2009); Baccarini, } \\
\text { Salm e Love (2004); Taylor (2006) }\end{array}$ \\
\hline \multirow{3}{*}{$\begin{array}{l}\text { Influências } \\
\text { Econômicas e } \\
\text { Ambientais }\end{array}$} & $\mathrm{F} 3 \mathrm{a}$ & Inflação e câmbio & \multirow{3}{*}{$\begin{array}{l}\text { Baccarini, Salm e Love (2004); } \\
\text { Grimsey e Lewis (2002); Taylor } \\
\text { (2006); Aundhe e Saji (2009); } \\
\text { Kloppenborg (2007); Dey (2002); } \\
\text { Mustafa e Al-Bahar (1991); } \\
\text { Kloppenborg (2007);Ewusi-Mensah e } \\
\text { Przasnyski (1991); Jarvenpaa e Ives } \\
\text { (1991); Wallace, Keil e Rai (2004, } \\
\text { 2004a); Lessard e Miller (2001); } \\
\text { Warkentin et al. (2009); Grimsey e } \\
\text { Lewis (2002); Dhar e Balakrishnnan } \\
\text { (2006); Taimour (2005) }\end{array}$} \\
\hline & $\mathrm{F} 3 \mathrm{~b}$ & $\begin{array}{l}\text { Ambiente } \\
\text { Organizacional }\end{array}$ & \\
\hline & $\mathrm{F} 3 \mathrm{c}$ & Leis e Regulamentos & \\
\hline \multirow[t]{2}{*}{$\begin{array}{l}\text { Planejamento do } \\
\text { Projeto }\end{array}$} & $\mathrm{F} 4 \mathrm{a}$ & $\begin{array}{l}\text { Metodologia a ser } \\
\text { utilizada }\end{array}$ & \multirow{2}{*}{$\begin{array}{l}\text { Kloppenborg (2007); Nieto-Marote e } \\
\text { Vila (2010); Wallace, Keil e Rai } \\
\text { (2004); Costa, Barros e Travassos } \\
\text { (2007); Elkington e Smallman (2000); } \\
\text { Brown (1996), Royce (1998), Goathan } \\
\text { (2009), Barros (2001), Taimor (2005) } \\
\text { e Pressman (2011) }\end{array}$} \\
\hline & $\mathrm{F} 4 \mathrm{~b}$ & Planejamento & \\
\hline
\end{tabular}

Fonte: Elaborada pelos autores (2016).

Estudos como os de Warkentin et al. (2009), Dey (2002); Elkington e Smallman (2000), Baccarini, Salm e Love (2004), Grimsey e Lewis (2002), Costa, Barros e Travassos (2007); Barki et al. (1993), Kemerer e Sosa (1988), McFarlan (1981), Schmidt et al. (2001); Wallace, Keil e Rai (2004, 2004a) defendem que a complexidade do projeto é crítica para o sucesso dos projetos, principalmente quando do uso de tecnologias pouco conhecidas, quando há instabilidade e a diversidade de requisitos. Os autores defendem que quando mais complexo o projeto, maior é a probabilidade de insucesso. Baseado nestas constatações, gerase a primeira hipótese $\left(\mathrm{H}_{1}\right)$ do estudo.

\section{$H_{1}$ : A Complexidade do Software impacta negativamente o sucesso dos projetos de desenvolvimento de softwares.}

Os autores Dey (2002), Kloppenborg (2007), Ewusi-Mensah e Przasnyski (1991), Jarvenpaa e Ives (1991), Wallace, Keil e Rai (2004a, 2004b), Lessard e Miller (2001), Warkentin et al. (2009), Mustafa e Al-Bahar (1991), Grimsey e Lewis (2002), Baccarini, Salm e Love (2004), Dhar e Balakrishnnan (2006) afirmam que fatores econômicos e ambientais, comumente fora do controle do time de projetos, podem ser decisivos para os resultados dos projetos. A estabilidade destes fatores 
permite um ciclo mais suave e controlável do projeto. Desta forma, forma-se a segunda hipótese $\left(\mathrm{H}_{2}\right)$ deste estudo:

\section{$\mathrm{H}_{2}$ : Influências Econômicas e Ambientais impactam o sucesso dos projetos de software.}

Nessa mesma linha, Kloppenborg (2007), Nieto-Marote e Vila (2010), Wallace, Keil e Rai (2004), Costa, Barros e Travassos (2007) e Elkington e Smallman (2000) entendem que o planejamento do projeto pode interferir na condução e no consequente resultado do projeto. Fatores como comunicação, definição de responsabilidades, definição de critérios de sucesso, uso de metodologias e de estimativas são fundamentais para o sucesso dos projetos. Estes estudos compõem a terceira hipótese $\left(\mathrm{H}_{3}\right)$ deste estudo:

\section{$\mathrm{H}_{3}$ : O Planejamento do Projeto impacta o sucesso dos projetos de software.}

Os pesquisadores Kloppenborg (2007), Nieto-Marote e Vila (2010), Wallace, Keil e Rai (2004), Costa, Barros e Travassos (2007), Elkington e Smallman (2000), Robey e Farrow (1982), Tait e Vessey (1988), Aundhe e Saji (2009) e Taylor (2006) apontam o relacionamento com os usuários do software como sendo um dos condicionantes para o sucesso do projeto de software. O comprometimento, as expectativas e os conflitos na equipe de desenvolvimento precisam ser bem geridos para evitar desgastes e resultados indesejados. Nesse mesmo sentido, Kloppenborg (2007), Abdel-Hamid e Madnick (1989), Alter e Ginzberg (1978), Jiang, Klein e Means (2000), Schmidt et al. (2001), Wallace, Keil e Rai (2004a, 2004b) Warkentin et al., (2009), Costa, Barros e Travassos (2007), Baccarini, Salm e Love (2004), Nieto-Marote e Vila (2010), Dhar e Balakrishnnan (2006) e Taylor (2006) afirmam que o envolvimento das partes interessadas nos projetos é fundamental para se atingir os resultados esperados. A falta de recursos, perdas de produtividade, falta de suporte e de comprometimento podem impactar o sucesso dos projetos. Estes trabalhos são a base para a quarta e última hipótese $\left(\mathrm{H}_{4}\right)$ deste estudo: 


\section{$\mathrm{H}_{4}$ : Envolvimento da Equipe e dos Usuários com o projeto impactam o sucesso dos projetos de desenvolvimento de softwares}

A seguir será apresentada a metodologia e o modelo estrutural resultante da revisão da literatura.

\section{Metodologia}

\subsection{Coleta de Dados}

Esse estudo foi realizado por meio de um levantamento com desenvolvedores, líderes e gerentes de projetos de softwares de empresas de desenvolvimento de softwares, no período entre junho e dezembro de 2016. Um questionário online contendo 21 perguntas fechadas (referentes aos fatores listados nas Tabelas 1 e 2) utilizando escala do tipo Likert de 5 pontos e duas questões fechadas utilizando escala dicotômica (opções sim e não) foi enviado para empresas com o perfil desejado da amostra identificadas por meio do cadastro da Federação Nacional das Empresas de Informática (FENAINFO, 2016) que abrange todas as regiões do Brasil. As empresas foram filtradas pela área de atuação e pelo porte. As empresas de infraestrutura de tecnologia da informação ( $\mathrm{TI}$ ) foram excluídas da amostra por não atenderem ao critério de desenvolvedora de software. Também foram excluídas da amostra as empresas classificadas como microempreendedor individual ou individual de responsabilidade limitada por terem porte reduzido para comportar equipes de projetos.

Foram enviados 387 e-mails com o link para o questionário online. Retornaram 77 respostas (taxa de retorno de 19,9\%). Das respostas, 61,3\% foram de empresas com mais de 100 empregados. Dos projetos desenvolvidos, 41,3\% utilizaram metodologias ágeis e $54,7 \%$ utilizaram metodologias tradicionais de desenvolvimento de software.

\subsection{Operacionalização do Modelo}

As bases teóricas e as hipóteses formuladas anteriormente formam o modelo estrutural apresentado na Figura 1. Cada uma das dimensões de fatores 
determinantes do sucesso dos projetos de desenvolvimento de software são constructos que supostamente impactam o sucesso dos projetos de software.

O modelo hipotético proposto foi investigado a partir da técnica de Modelagem de Equações Estruturais (PLS-SEM - Partial Least Squares Structural Equation Modeling) utilizando o software SmartPLS3 (Ringle; Wende; Becker, 2015). Todos os indicadores são de caráter formativo.

Os 14 fatores apresentados na Tabela 2 são os indicadores dos constructos formativos, que foram formados pelas dimensões levantadas na literatura. Estes formam o modelo estrutural proposto e apresentado na Figura 1, assim como cada uma das hipóteses levantadas na literatura.

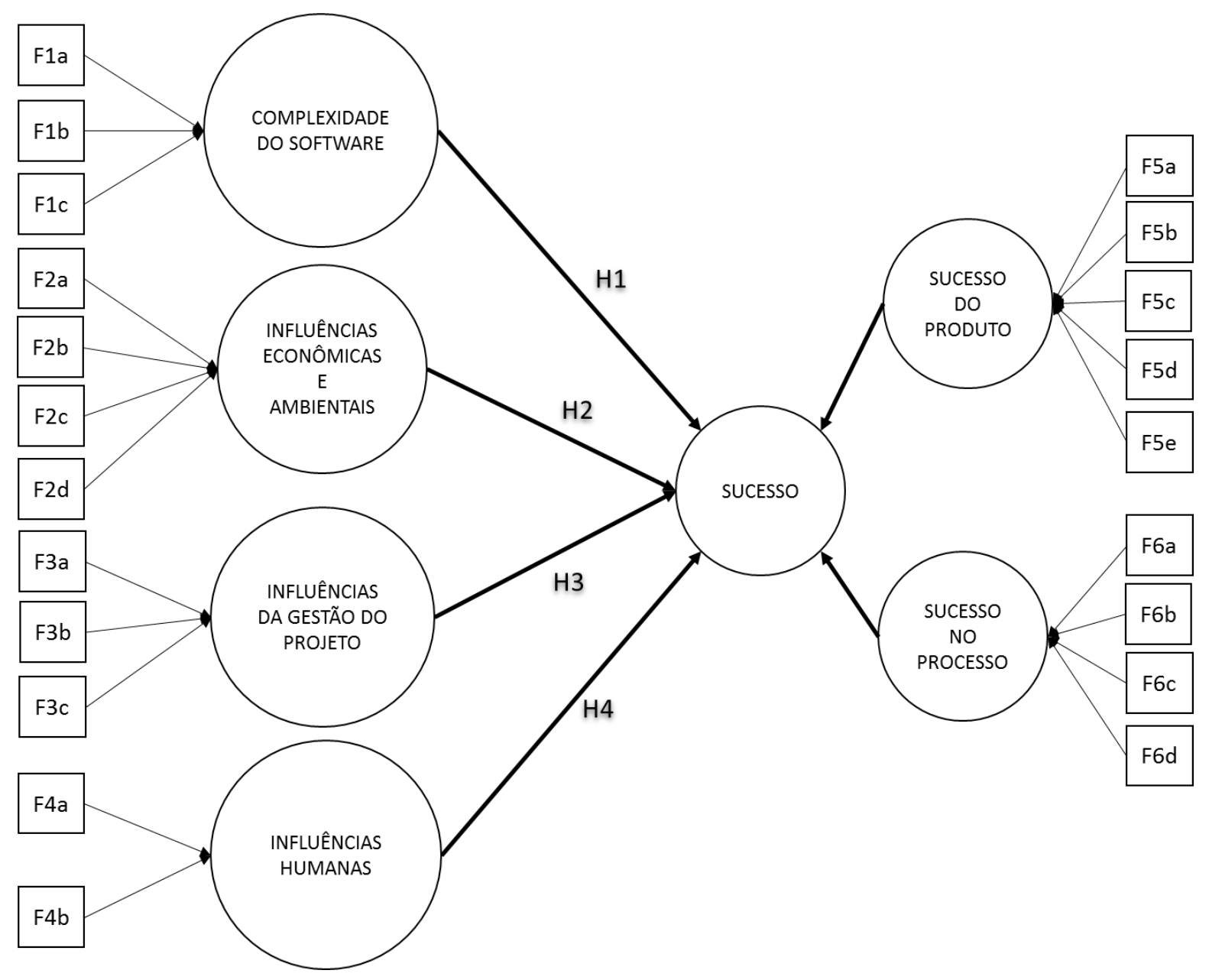

Figura 1

Modelo estrutural proposto

Fonte: Elaborada pelos autores(2017) 


\section{Resultados}

Segundo Hair et al. (2014, p. 20) o tamanho da amostra submetida a um modelo de equações estruturais deve ter pelo menos 10 vezes o número de indicadores do constructo formativo com o maior número de indicadores. O modelo proposto possui o constructo Produto com cinco indicadores, sendo a amostra mínima necessária de 50 casos. Com o objetivo de aumentar a precisão no que tange ao tamanho da amostra, foi adicionalmente realizado um cálculo de tamanho de amostra a partir do software GPower versão 3.1, que é uma ferramenta de uso livre para análise de uma variedade de testes estatísticos (FAUL et al., 2009), indicada por Hair et al., (2014). Ainda segundo a indicação de Hair, et al. (2014), para avaliar o tamanho da amostra devem ser observados dois parâmetros: o poder do teste (1- $\beta$ err prob) e o tamanho do efeito ( $\{2$ ) e recomendam, para pesquisas em ciências sociais, o poder de 0,80 e f2 de 0,15 . No uso do software foram considerados 5 preditores. O GPower indicou que a amostra precisa ter mais de 43 respondentes, que é um valor inferior ao processo simplificado sugerido por Hair, et al., (2014).

A pesquisa realizada obteve um total de 78 respostas válidas, tendo, portanto, cumprindo este critério.

\subsection{Modelos de Mensuração}

Todos os constructos são de caráter formativo, ou seja, os indicadores cobrem todo o escopo de cada uma das variáveis latentes. O caráter formativo é corroborado pelas condições defendidas por Jarvis, Scott e Podsakoff (2003) que incluem: a) os indicadores definem as características do constructo; b) mudanças nos indicadores causam mudanças no constructo; c) mudanças no constructo não causam mudanças nos indicadores; d) os indicadores não compartilham o mesmo tema; e) a eliminação de um indicador altera a o conceito do domínio; f) a mudança em um indicador não necessariamente provoca mudanças em outro indicador e; g) os indicadores não têm o mesmo antecedente e consequente.

Por serem formativos, os modelos de mensuração precisam ser validados para que não existam multicolinearidades segundo Hair, Ringle e Sarstedt (2011). A multicolinearidade é avaliada a partir do Fator de Inflação da Variância (VIF) que deve ser inferior a 5,0 (Hair et al, 2011). Todos os indicadores atenderam aos 
critérios, sendo o indicador F2c com o maior valor de VIF (1.139) e F1b com o menor valor (1.049).

A relevância e significância dos pesos foram avaliadas a partir da função bootstrapping com 5.000 interações. $O$ indicador complexidade dos requisitos ( $F 1 b$ ) não apresentou peso fatorial significante acima de 0,50 , porém, para manter a consistência do constructo formativo teórico, optou-se por mantê-lo no modelo (Hair et al., 2014). Os demais indicadores, ou apresentaram peso fatorial acima de 0,50, ou foram significantes a 0,05 no peso fatorial, apresentando importância absoluta e/ou relativa (Hair et al., 2014) e, por este motivo, foram mantidos no modelo.

\subsection{Análise do Modelo Estrutural}

Após a verificação dos modelos de mensuração, o modelo estrutural foi analisado através dos coeficientes de caminho apresentados na Tabela 3. Apenas o coeficiente de caminho de Influências Econômicas e Ambientais para Sucesso não foi significante. Os coeficientes dos constructos Complexidade do Software foi significante a 0,05 e Envolvimento da Equipe e dos Usuários e Planejamento do Projeto a 0,10 (95 e 90\% de nível de confiança respectivamente).

\section{Tabela 3}

Coeficientes de Caminho

\begin{tabular}{lcc}
\hline Constructos & $\begin{array}{c}\text { Coeficiente de } \\
\text { Caminho }\end{array}$ & p-valor \\
\hline Complexidade do Software -> Sucesso & $-0,411$ & 0.000 \\
Influências Econômicas e Ambientais -> Sucesso & $\mathbf{- 0 , 0 3 0}$ & $\mathbf{0 . 7 4 5}$ \\
Planejamento do Projeto -> Sucesso & 0,206 & 0.071 \\
Envolvimento da equipe e dos usuários -> Sucesso & 0,230 & 0.089 \\
\hline Fonte: Elaborada pelos autores (2016). & &
\end{tabular}


A Figura 2 mostra os resultados da análise do modelo estrutural proposto.

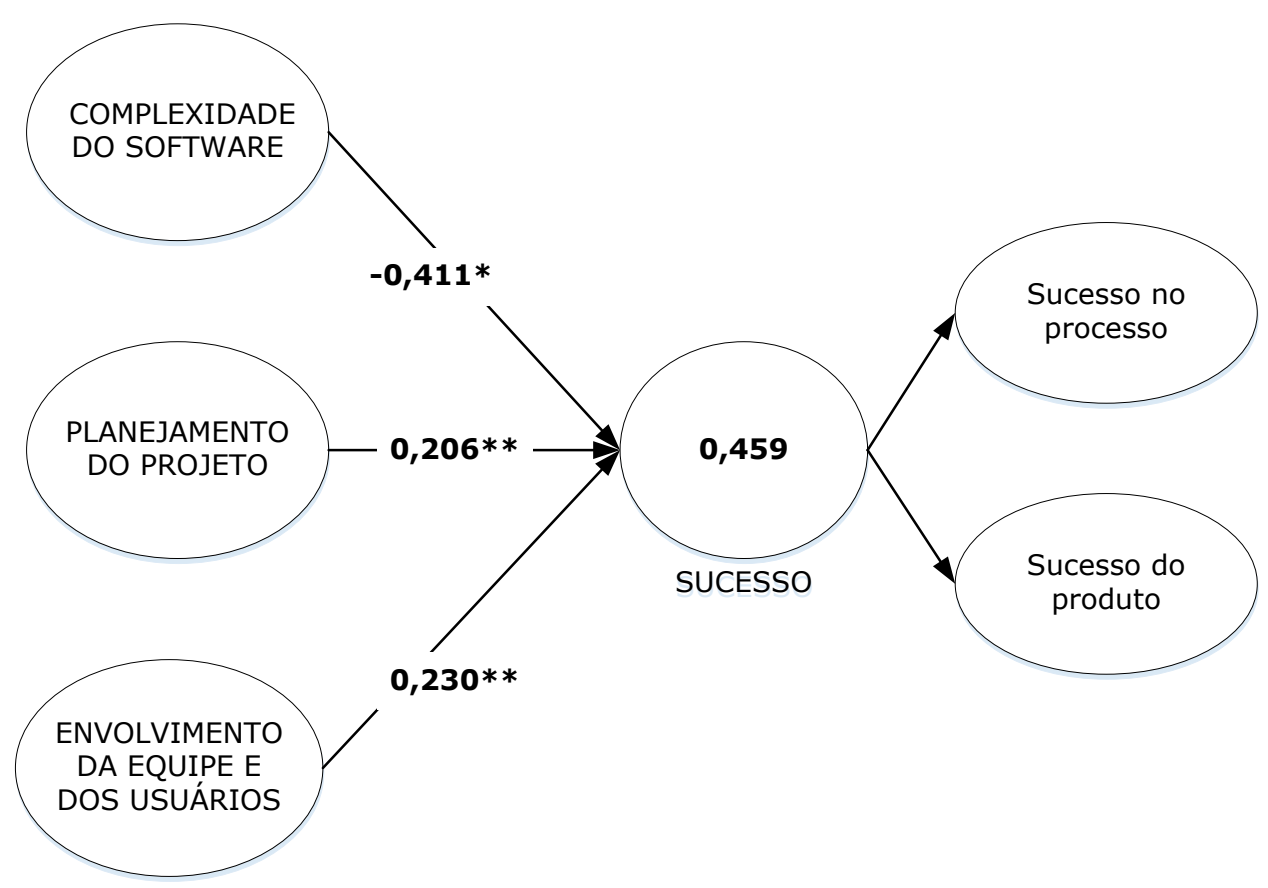

Figura 2

Resultado do modelo completo após a análise Fonte: Elaborada pelos autores (2016).

Legenda: * $p$-valor menor que $0,05,{ }^{*}$ p-valor menor que 0,10

O coeficiente de determinação $R^{2}$ do constructo Sucesso foi de 0,459 . Os fatores determinantes do sucesso de projetos de software são capazes de explicar 45,9\% da variância do constructo Sucesso dos projetos de software, uma capacidade explicativa considerada aceitável para pesquisas em ciências sociais (Hair et al., 2011).

A Tabela 4 apresenta os resultados finais das hipóteses propostas. Os coeficientes de caminho significativos suportam as hipóteses do estudo. Apenas a hipótese relacionada ao constructo Influências Econômicas e Ambientais foi rejeitada

\section{Tabela 4}


Avaliação das Hipóteses

\begin{tabular}{l|l|l}
\hline \multicolumn{1}{c|}{ Hipóteses } & Resultado \\
\hline $\mathrm{H}_{1}$ & $\begin{array}{l}\text { A Complexidade do Software impacta negativamente o sucesso dos projetos } \\
\text { de desenvolvimento de software. }\end{array}$ & Suportada \\
\hline $\mathrm{H}_{2}$ & $\begin{array}{l}\text { Influências Econômicas e Ambientais impactam o sucesso dos projetos de } \\
\text { desenvolvimento software. }\end{array}$ & Rejeitada \\
\hline $\mathrm{H}_{3}$ & $\begin{array}{l}\mathrm{O} \text { Planejamento do Projeto impacta o sucesso dos projetos de } \\
\text { desenvolvimento de software. }\end{array}$ & Suportada \\
\hline $\mathrm{H}_{4}$ & $\begin{array}{l}\text { Envolvimento da Equipe e dos Usuários com o projeto impacta o sucesso dos } \\
\text { projetos de desenvolvimento de software. }\end{array}$ & Suportada \\
\hline
\end{tabular}

Fonte: Elaborada pelos autores (2016).

Um teste de comparação entre médias foi realizado para garantir que os coeficientes de caminho não sejam iguais e que pudessem ser comparados como tendo pesos diferentes no sucesso dos projetos de software. Os erros padrão gerados pelas 5.000 interações da técnica bootstrapp foram utilizados no teste de diferenças de médias para avaliar se os valores dos coeficientes de caminho podem ser considerados diferentes e possíveis de serem comparados. Todos os coeficientes de caminho tiveram a hipótese de médias iguais rejeitadas, sendo possível, portanto, de serem comparadas.

Com o objetivo de analisar as dimensões de sucesso de forma individualizada e entender qual condicionante tem maior influência em uma determinada dimensão de sucesso, foram executados dois novos cenários para as hipóteses propostas. Os cenários envolvem o teste do mesmo modelo para cada uma das dimensões de sucesso de forma segmentada: do Produto e no Processo de Desenvolvimento de Software. Para tal, os mesmos procedimentos de validação e análise foram executados e atendidos.

Para o primeiro cenário, Sucesso no Processo de Desenvolvimento do Software, os resultados mostraram um $R^{2}$ de 0,307 . Os coeficientes de caminhos significantes foram dos constructos Complexidade do Software e Planejamento do Projeto (p-valor: 0,020 e 0,091 respectivamente). O constructo Envolvimento da Equipe e dos Usuários e Influências Econômicas e Ambientais não se mostraram significantes. A Figura 3 ilustra os resultados obtidos. 


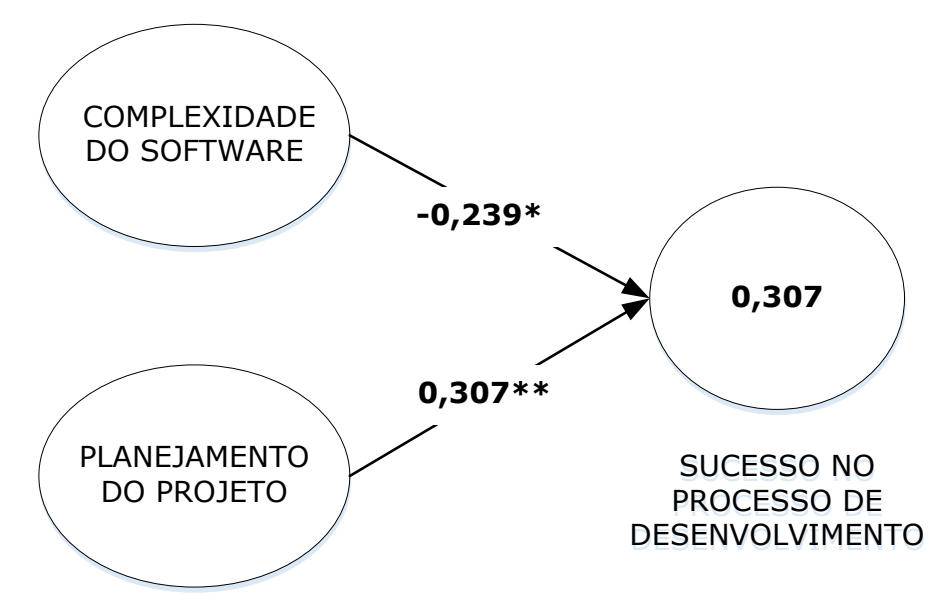

\section{Figura 3}

Resultado do modelo para sucesso no processo de desenvolvimento Fonte: Elaboração Própria (2016)

Legenda: ${ }^{*} p$-valor menor que $0,05,{ }^{* *} p$-valor menor que 0,10

$\mathrm{Na}$ análise do segundo cenário, com apenas o Sucesso do Produto $\circ \mathrm{R}^{2}$ resultante foi de 0,454 e apenas o coeficiente de caminho Complexidade do Software foi significativo $(0,000)$. A Figura 4 mostra o modelo resultante da análise.

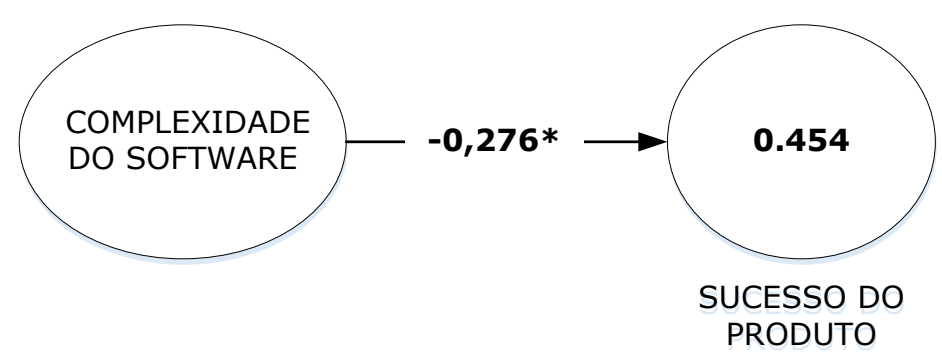

Figura 4

Resultado do modelo para sucesso do produto de software Fonte: Elaboração Própria (2016)

Legenda: ${ }^{\star} p$-valor menor que $0,05,{ }^{*}$ p-valor menor que 0,10

\section{Discussões}

A hipótese $\mathrm{H}_{1}$ - A Complexidade do Software impacta negativamente o sucesso dos projetos de desenvolvimento de software não foi rejeitada. Pode-se afirmar, portanto, que a complexidade impacta o sucesso dos projetos de software. Como o coeficiente de caminho do constructo Complexidade do Software foi negativo $(-0,411)$, quanto maior a complexidade do software, menor é o sucesso do projeto, o que corrobora as pesquisas de Warkentin et al. (2009), Dey (2002), Costa, Barros e Travassos (2007), Schmidt et al. (2001) e Wallace, Keil e Rai (2004, 2004a). Uma maior complexidade leva a uma maior dificuldade no desenvolvimento, 
seja originada pelos requisitos, seja originada pelo nível de inovação ou de novas tecnologias envolvidas.

A hipótese $\mathrm{H}_{2}$ - Influências Econômicas e Ambientais impactam o sucesso dos projetos de desenvolvimento software foi rejeitada, ou seja, não foi possível demonstrar que Influências Econômicas e Ambientais ligadas a mudanças em leis, câmbio, inflação, defeitos em equipamentos, cultura organizacional impactam no sucesso dos projetos de software. As Influências Econômicas e Ambientais tradicionalmente aparecem em pesquisas de projetos de infraestrutura como construção civil, por exemplo (Dey, 2002; Mustafa \& Al-Bahar, 1991; Grimsey \& Lewis, 2000). Como projetos de softwares normalmente não envolvem aquisições ou o uso intenso de matérias primas e equipamentos, os impactos econômicos e ambientais acabam sendo menores. Estas influências estão muito mais presentes em projetos de infraestrutura e, conforme mostra a Tabela 2, nenhum dos fatores levantados para esta dimensão é específico para projetos de software (DEY, 2002).

A hipótese $\mathrm{H}_{3}-\mathrm{O}$ Planejamento do Projeto impacta o sucesso dos projetos de desenvolvimento de software não foi rejeitada. Conclui-se, portanto, que o planejamento influencia o sucesso dos projetos de software. As metodologias aplicadas ao projeto e o planejamento correto podem ser determinantes para 0 sucesso dos projetos. Um planejamento ruim leva a cronogramas e orçamentos irreais, além da falta de marcos, entregas e critérios de sucesso estabelecidos o que pode levar o projeto ao fracasso. Além disso, a falta de processos adequados de planejamento pode influenciar os resultados do projeto. Estes achados estão alinhados com as pesquisas de Kloppenborg (2007), Nieto-Marote \& Vila (2010), Wallace et al. (2004), Costa, Barros e Travassos (2007) e Elkington e Smallman (2000).

A hipótese $\mathrm{H}_{4}$ - Envolvimento da Equipe e dos Usuários com o projeto impacta o sucesso dos projetos de desenvolvimento de software não foi rejeitada. O envolvimento e comprometimento dos usuários é um dos fatores que mais aparecem em estudos sobre projetos de software (Wallace et al, 2004), usuários não favoráveis ao software podem não cooperar e prejudicar o desenvolvimento deste, além disso, a falta de comprometimento, conflitos, cooperação e motivação da equipe do projeto podem prejudicar os resultados dos projetos. Estes achados estão em linha com os estudos de Wallace et al. (2004a, 
2004b) Warkentin et al. (2009), Costa, Barros e Travassos (2007), Baccarini, Salm e Love (2004) e Nieto-Marote e Vila (2010).

A partir dos coeficientes de caminho listados na Tabela 1 e na Figura 2 é possível observar evidências de que a Complexidade do Software $(-0,411)$, Planejamento do Projeto $(0,206)$ e Envolvimento da Equipe e dos Usuários $(0,230)$ tem diferentes impactos no sucesso dos projetos de software. A Complexidade do Software envolve fatores como requisitos complexos e estáveis, inovação, tecnologias emergentes, integrações e padrões de desenvolvimento. Estes aspectos, segundo o resultado da pesquisa, tem um maior peso no resultado dos projetos e devem, portanto, serem observados e monitorados com cuidado com o objetivo de influenciar positivamente os resultados dos projetos de desenvolvimento de softwares. Esse resultado evidencia a importância e o peso destes aspectos no processo de desenvolvimento.

O Planejamento do Projeto foi o segundo constructo com maior peso relativo no sucesso dos projetos. Envolve aspectos como a metodologia utilizada, precisão das estimativas, comunicação e definição de responsabilidades. Um achado interessante desta pesquisa empírica foi que a Planejamento do Projeto, ao contrário do que várias pesquisas sobre gestão de projetos afirmam (Elkington \& Smallman, 2000; Wallace, Keil \& Rei, 2004), não é o principal aspecto que determina os resultados dos projetos de software nas empresas pesquisadas no Brasil.

Tanto a Planejamento do Projeto quanto o Envolvimento da Equipe e dos Usuários tiveram coeficientes de caminho positivos indicando, portanto, que, melhorias nestes fatores trazem impactos positivos para o sucesso do projeto. Em suma, a Complexidade do Software é inversamente proporcional ao sucesso do projeto tendo um impacto negativo, enquanto a Planejamento do Projeto e o Envolvimento da Equipe e dos Usuários tem impactos positivos nos resultados dos projetos.

A análise segmentada das dimensões de sucesso levantados na literatura mostram resultados interessantes, quando apenas o Sucesso do Processo de Desenvolvimento é envolvido na análise. O Sucesso no Processo de Desenvolvimento envolve apenas critérios tradicionais de medida de sucesso de projetos como: custo, prazo, qualidade e escopo. Para estes critérios, em projetos de software, apenas os constructos Complexidade do Software e Planejamento do Projeto influenciam seu resultado. O Planejamento do Projeto um peso maior, 
alinhado com o que afirmam vários teóricos da Gestão de Projetos que afirmam que o planejamento é fundamental para o sucesso dos projetos (Kerzner, 2010). Os resultados mostram indícios de que, ao contrário do modelo de sucesso global, o Envolvimento da Equipe e dos Usuários não é um fator condicionante com impacto direto para o processo de desenvolvimento. Para este cenário, apenas as hipóteses $\mathrm{H}_{1}$ e $\mathrm{H}_{3}$ foram suportadas.

Quando apenas o Sucesso do Produto é envolvido na análise, apenas o constructo Complexidade do Software se mostra significante, mostrando indícios de que satisfação quanto ao produto em aspectos como tempo de resposta, usabilidade e manutenabilidade são influenciados apenas pelo nível de complexidade do Software e não dependem de fatores como Envolvimento da Equipe e Usuários e nem mesmo da qualidade do Planejamento do Projeto. Para este cenário, apenas a Hipótese $H_{1}$ foi suportada. Este resultado corrobora algumas metodologias de gestão de projetos que afirmam que a gestão e o planejamento do projeto são desvinculados do produto ou serviço que está sendo entregue (PMI, 2013).

Um ponto adicional que se destaca é que o Envolvimento da Equipe e Usuários somente se torna significativo quando ambas as dimensões de sucesso são analisadas ao mesmo tempo. Os resultados sugerem que o envolvimento das partes interessadas é um componente de influência abrangente e indireta e que não tende a influenciar pontualmente o produto ou o processo, e sim os resultados do projeto de maneira integral.

\section{Considerações Finais}

Este estudo propôs um modelo estrutural que relaciona os determinantes do sucesso de projetos ao sucesso de projetos de software. A partir de uma pesquisa empírica, foi possível responder as seguintes perguntas de pesquisa: Quais são os fatores condicionantes do sucesso de projetos de desenvolvimento de software? Quais fatores tem maior influência no sucesso dos projetos de software?

Os condicionantes suportados pela pesquisa empírica foram aqueles relacionados aos domínios de Complexidade do Software, Planejamento do Projeto 
e o Envolvimento da Equipe e dos Usuários. Apenas a Influência dos Fatores Econômicos e Ambientas não se mostrou significativo em nenhum dos cenários apresentados não sendo possível obter evidências de que estes fatores impactam os resultados dos projetos de software.

O nível de impacto de cada fator foi demonstrado a partir dos coeficientes de caminho. Os resultados mostraram que a Complexidade do Software tem um maior peso nos resultados dos projetos, seguidos por Planejamento do Projeto e Envolvimento da Equipe e dos Usuários.

Estes resultados trazem uma contribuição para o meio acadêmico e empresarial ao mostrarem indícios de que alguns fatores são mais importantes que outros e que estes podem ser decisivos no sucesso dos projetos de desenvolvimento de softwares. Seguindo uma ordem de prioridade devem, portanto, ser trabalhados e monitorados com atenção para que os projetos atinjam os resultados desejados. Procedimentos de gestão de risco podem ser aplicados a estes fatores com o objetivo de mitigar e até eliminar problemas antes e durante a execução dos projetos.

Os critérios (atributos) de medição do sucesso de projetos de software foram também estabelecidos neste artigo. Estes podem ser utilizados como referência em trabalhos futuros que envolvam investigar o sucesso em projetos de software.

Os aspectos relacionados a Planejamento do Projeto podem ser afetados dependendo da metodologia ou estratégia de desenvolvimento selecionada para o projeto. O desenvolvimento tradicional, utilizando as técnicas de gestão de projetos tem demonstrado resultados diferentes de técnicas inovadoras como 0 desenvolvimento ágil, por exemplo, e isso pode interferir de forma significativa nos resultados, sendo isso uma limitação desta pesquisa.

Como trabalhos futuros, sugere-se que sejam analisados os efeitos moderadores da metodologia aplicado aos projetos com o objetivo de entender como eles interferem nos resultados dos projetos.

\section{Referências}

Abdel-Hamid, T., \& Madnick, S. E. (1989). Lessons learned from modeling dynamics of software development. Management of Computing, 32(12), 1426- 1465.

Alter, S., \& Ginzberg, M. (1978). Managing uncertainty in MIS implementation. Sloan Management Review, 20(1), 23-31. 
Amrit, C., Hillegersberg, J. V., \& Kumar, K. (2012). Identifying coordination problems in software development: finding mismatches between software and project team structures. Cornwell University.

Anantatmula, V. (2015). Strategies for enhancing project performance. Journal of Management in Engineering, 31(6), 1-8.

Atkinson, R. (1999). Project management: cost, time and quality, two best guesses and a phenomenon, its time to accept other success criteria. International Journal of Project Management, 17(6), 337-342.

Aubry, M. Hobbs, B. (2011). A fresh look at the contribution of project management to organizational performance. Project Management Journal, 42(1), 3-16.

Aundhe, M., \& Saji, M. (2009). Risks in offshore IT outsourcing: A service provider perspective. European Management Journal, 27(6), 418-428.

Baccarini, D., Salm, G., \& Love, P. (2004). Management of risks in information technology projects. Industrial Management and Data System, 104(4), 286-295.

Barki, H., Riverd, S., \& Talbot, J. (2003). Toward an assessment of software development risk. Journal of Management Information Systems, 10(2), 203-225.

Barros, M. O. (2001). Gerenciamento de projetos baseado em cenários: uma abordagem de modelagem dinâmica e simulação. (Tese de Doutorado. Universidade Federal do Rio de Janeiro, Rio de Janeiro, RJ, Brasil).

Bredillet, C.N. (2010). Blowing hot and cold on project management. Projet Management Journal, 41(3), 4-20.

Brown, N. (1996). Industrial-strength management strategies. IEEE Software, 13(4), 94-103.

Bouer, R., \& Carvalho, M. M. (2005). Metodologia singular de gestão de projetos: condição suficiente para a maturidade em gestão de projetos? Produção, 15(3), 347-361.

Chan, A.P.L. (2001). Key performance indicators for measuring construction success Benchmarking, 11(2), 203-221.

Charette, R.N. Why software fails. 2005. (2005). IEEE Spectrum. Recuperado em 01, maio, 2016 de http://spectrum.ieee.org/computing/software/why-softwarefails.

Cleland, D.I. (1999). Project management: strategic design and implementation (3a ed). New York: McGraw ill.

Cooke-Davies, T. (2002). The real success factors on projects. International Journal of Project Management, 20(3), 185-190. 
Costa, H.R., Barros, M. O., \& Travassos G.H. (2007). Evaluating software project portfolio risks. Journal of Systems and Software, 80 (1), 16-31.

Davis, K. (2014). Different stakeholder groups and their perceptions of project success. International Journal of Project Management, 2 (32), 189-201.

DeLone, W. H., \& McLean, E. R. (1992). Information Systems Success: The Quest for the Dependent Variable. Information Systems Research, 3(1), 60-95.

Dey, P K. (2002). Project risk management: a combined analytic hierarchy process and decision tree analysis approach. Cost Engineering Journal American Associate of Cost Engineering International (AACEI), 44(3), 13-26.

De Wit, A. (1988). Measurement of project success. International Journal of Project Management, 6(3), 164-170.

Dhar, S., \& Balakrishnan, B. (2006). Risks, benefits, and challenges in global it outsourcing: perspectives and practices. Journal of Global Information Management, 14(3), 39-68.

Dvir, D., Lipovetsky, S., \& Tisher, A. (1998). In search of project classification: a nonuniversal approach to project success factors. Research Policy, 27(9), 917-935, 1998.

Elkington, P., \& Smallman, C. (2000). Managing project risk: a case study from the utilities sector. International Journal of Project management, 20(1), 49-57.

Ewusi-Mensah, K. Przasnyski, Z. (1991). On information systems project abandonment: an exploratory study of organizational practices. MIS Quarterly, 15(1), 67-86.

Faul, F., et al. (2009). Statistical power analyses using G*Power 3.1: tests for correlation and regression analyses. Behavior Research Methods, 41(4), 11491160.

FENAINFO. Federação Nacional das Empresa em Informática. Recuperado em 15, ago., 2016 de http://fenainfo.org.br/.

Freeman, M, Beale, P. (1992). Measuring project success. Project Management Journal, 23(1), 8-17.

Goathan, R. (2009). The story behind the high failure rates in the IT sector. Calleam Consulting Ltd.

Gray, R. J. (2001). Organisational climate and project success. International Journal of Project Management, 10(2), 103-109.

Grimsey, D., \& Lewis K. (2002). Evaluating the risks of public private partnerships for infrastructure projects. International Journal of Project Management, 20(2), 10718. 
Hair Jr, J. F. et al. (2014). A primer on partial least squares structural equation modeling (PLSSEM). California: Sage.

Hair, J.F., Ringle, C.M., \& Sarstedt, M. (2011). PLS-SEM: indeed, a silver bullet, in: Journal of Marketing Theory and Practice (JMTP), 19(2), 39-152.

Ika, L. (2009). Project success as a topic in project management journals. Project manager Journal, 40(4), 6-19.

Jarvenpaa, S.L., \& Ives, B. (1991). Executive involvement and participation in the management of information technology. MIS Quarterly, 15(2), 204-227.

Jarvis C. B., Scott S. B., \& Podsakoff, P. M. (2003). A critical review of construct indicators and measurement model misspecification in marketing and consumer research. Journal of Marketing Research, 60(3), 15-32.

Jiang, J.J., Klein, G., \& Means, T.L. (2000). Project risk impact on software development team performance. Project Management Journal, 31(4), 19-26.

Kaur, R., \& Sengpta, J. (2011). Software process models and analysis on failure of software development projects. International Journal of Scientific and Engineering Research, 2(5), 1-4.

Kemerer, C., \& Sosa, G. (1988). Barriers to successful strategic information systems. Planning Review, 16(5), 20-23.

Kerzner, H. (2010). Gestão de Projetos: As melhores práticas. São Paulo: Artmed.

Kloppenborg, T. (2007). IT project risk factors: The project management professional's perspective. Journal of Computer Information Systems, 47(4), 6169.

Kunz, P. (1989). Software management issues. Computer Physics Communications. 57(1), 191-197.

Lessard, R., \& Miller, R. (2001). Understanding and managing risks in large engineering projects. MIT Sloan Working Paper, 4214(1), 1-17.

Lim, C. S., \& Mohamed, M. Z. (1999). Criteria of project success: an exploratory reexamination. International Journal of Project Management, 17(4), 243-248.

McFarlan, F. W. (1981). Portfolio approach to information systems. Harvard Business Review, 59 (1), 142-150.

Mills K., \& Mercken R. (2002). Success factors regarding the implementation of ICT investment projects. International Journal of Production and Economics, 80(1), 105-117. 
Moura, R. (2015). O Insucesso de Projetos de Desenvolvimento de Software e Fatores Influenciadores. Revista Científica Cet-Faesa, 7(1), 59-63.

Moura, R., \& Carneiro, T. (2015) O DNA do gerente de projetos: proposição de um modelo teórico parcimonioso usando algoritmo genético. Anais do $4^{\circ}$ Singep Simpósio Internacional de Gestão de Projetos, Inovação e Sustentabilidade, São Paulo, SP, Brasil.

Nieto-Marote, A., \& Vila, F. (2010). A fuzzy approach to construction project risk assessment. International Journal of Project Management, 32(10), 847-857.

Mustafa, M., \& Al-Bahar J. (1991). Project risk analytic assessment using the hierarchy process. IEEE Transactions on Engineering Management, 38(1), 4652.

Prado, D.S. (1999). Gerência de projetos em tecnologia de informação. (Série Gerência de Projetos, v. 5). Belo Horizonte: Editora de Desenvolvimento Gerencial.

Pressman, R.S. (2011). Engenharia de software (7a ed.). Mcgrraw Hill-Artmed.

Project Management Institute (PMI). (2013). A Guide to the project management body of knowledge - Pmbok® (Guide 5 Edition). Pennsylvania-USA.

Project Management Institute (PMI). (2015). Capturing value of project management. Recuperado em 07 maio, 2016 de https://www.pmi.org/ /media/PDF/learning/pulse-of-the-profession-2015.ashx.

Reel, J. S. (1999). Critical success factors in software projects. IEEE Software: Xplore, 740(99), 18-23.

Ringle, C. M., Wende, S., \& Becker, J.M. (2015). SmartPLS 3. Boenningstedt: SmartPLS GmbH. Disponível em http://www.smartpls.com.

Robey, D., \& Farrow, D. L. (1982). User involvement in information system development: a conflict model and empirical test. Management Science, 28(1),75-83.

Royce, W. (1998). Software project management: a unified framework. The AddisonWesley.

Schilling, M., \& Hill, C. (1998). Managing the new product development process: strategic imperatives. Academy of Management Executive, 12(3), 67-81.

Schmidt, R., Lyytinen, K., Keil, M., \& Cule, P. (2001). Identifying software project risks: an international delphi study. Journal of Management Information Systems, 17(4), 5-36.

Standish Group, T. (2015) The chaos report. The Standish Group Report. Recuperado em 13 set., 2015 de https://www.projectsmart.co.uk/whitepapers/chaos-report.pdf. 
Taimor, Al N. (2005). Why IT projects fail. The project perfect White Paper Collection. Sidney, Austrália. Recuperado em 15 ago., 2015 de www.projectperfect.com.au.

Taylor, H. (2006). Critical risks in outsourced it projects: the intractable and unforeseen. Communication of the ACM, 49(11), 75-79.

Tait, P., \& Vessey, I. (1988). The effect of user involvement on system success: a contingency approach, MIS Quarterly, 12(1), 91-107.

Wallace, L., Keil, M., \& Rai, A. (2004). How software project risk affects project performance: an investigation of the dimensions of risk and an exploratory model. Decisions Sciences, 35(2), 289-321.

Wallace, L., \& Keil, M. (2004). Software project risks and their effect on outcomes. Communication of Theach, 47(4), 68-73.

Warkentin, M., Moore, R.S., Bekkering, E., \& Jonhston, A.C. (2009). Analysis of systems development project risks: an integrative network. ACM SIGMIS Database, 40(2), 927-937. 